\title{
Dynamic Trunk Control Influence on Run-to-Cut Maneuver: A Risk Factor for ACL Rupture
}

\author{
By: Steve Jamison ${ }^{1}$
}

\section{INTRODUCTION}

Anterior cruciate ligament (ACL) rupture is one of the most common knee injuries for athletes competing in field and court sports. Hewett et al. reported that more than 2,200 ACL ruptures are expected to occur in female collegiate athletes each year and that a conservative estimate for orthopaedic care for this group alone would amount to over \$37 million [1]. When one considers that these injuries occur to males and females participating in sport at all levels from junior to elite to adult recreational, the total direct medical costs are several fold higher. The consequences of ACL rupture also go far beyond the medical costs of initial treatment. Injured athletes also face the potential loss of the remainder of the season, long-term disability, increased risk of osteoarthritis, elevated pain levels during sport, and, for collegiate athletes, lowered academic performance and possible loss of a scholarship [2-3].

Approximately 70\% of ACL ruptures are non-contact in nature, meaning that they occur without a direct blow to the leg by an object or opposing player, and most of these occur during sudden changes in direction and pivoting [4-6]. Previous studies have shown that both knee abduction moments and tibial internal rotation moments strain the ACL and that these moments have an interaction effect at physiologic load levels, creating strains approaching the reported

\footnotetext{
${ }^{1}$ I would also like to gratefully acknowledge my advisor Ajit Chaudhari, PhD and Jeff Pan, PhD for their significant contributions to this work.
} 
range of ACL rupture [7-10]. One prospective cohort study has shown that peak knee abduction moment during a drop-jump activity could predict ACL rupture with 73\% specificity and 78\% sensitivity in a population of 205 female athletes, further implicating increased peak knee abduction moments as a factor in ACL injury risk [11].

A study by Zazulak et al. found that deficits in lateral trunk control, measured using an isolated trunk perturbation test, were correlated with ACL injury incidence [12]. During this test the subject's pelvis was secured to isolate the torso and upper extremity. The subject then isometrically pulled against cable until the cable was randomly released. Those subjects with increased angular deviation of the trunk after the release were at a greater risk of ACL injury than those that had better neuromuscular control of the trunk. While this study indicates that a relationship does exist between trunk control and ACL injury risk, it is not able to justify a rationale for the relationship. It's possible that this relationship is merely coincidental and athletes who exhibit increased trunk control are also better conditioned, making this population less apt to sustain an ACL injury in general. Another possibility is that control of the trunk in the isolated trunk perturbation test is a good indication of dynamic trunk control during certain cutting and pivoting tasks and that the resulting trunk position directly influences the dynamic loading of the knee.

The goal of this study was to determine if dynamic control of the trunk during a run-tocut maneuver was correlated with the dynamic loading of the knee during the same activity. It was hypothesized that increased torso motion away from the cutting direction would have a significant, positive association with both knee abduction moments and tibial internal rotation moments. 


\section{METHODS}

Subjects. Thirty healthy subjects (15 male, 15 female; $1.73 \pm 0.09 \mathrm{~m} ; 68.5 \pm 12.4 \mathrm{~kg} ; 26.4 \pm 6.9 \mathrm{yrs}$ ) participated in this study after providing IRB approved consent. Subjects had no prior history of lower extremity surgery or serious injury nor did they have a previous history of open abdominal surgery. All subjects were pain free at the time of testing and were fit enough to comfortably jog for more than 10 minutes.

Unanticipated Run-to-Cut Maneuver: Subjects started on a pressure sensitive mat then jogged 3 steps at a self-selected pace before planting their dominant foot within a target area define by a Bertec force plate 40cm wide by 60cm long [Bertec 4060-10; Bertec Corp; Columbus, Ohio] embedded in the floor to record $(1500 \mathrm{~Hz})$ the ground reaction forces acting upon the foot when landing. Just before the subjects planted for their cut, an arrow pointing in one of two directions would illuminate, instructing the subjects to either continue running straight or to perform the cut. The direction of the arrow was chosen at random. A successful cut was one in which the plant foot was completely within the $40 \mathrm{~cm}$ wide by $60 \mathrm{~cm}$ long target area and the subject's change of direction took him/her over top of a line drawn on the floor at a $45^{\circ}$ angle to the approach direction, originating at the middle of the force plate. The distance between the pressure mat and the plant foot target as well as the time between when the subject left the pressure mat and when an arrow would be illuminated were adjusted for each subject to account for stride length, speed, and reaction time differences between subjects. Dominant foot was established as the foot the subject would prefer to use to kick a ball. Figure 1 shows the laboratory set-up for the unanticipated cutting maneuver. 


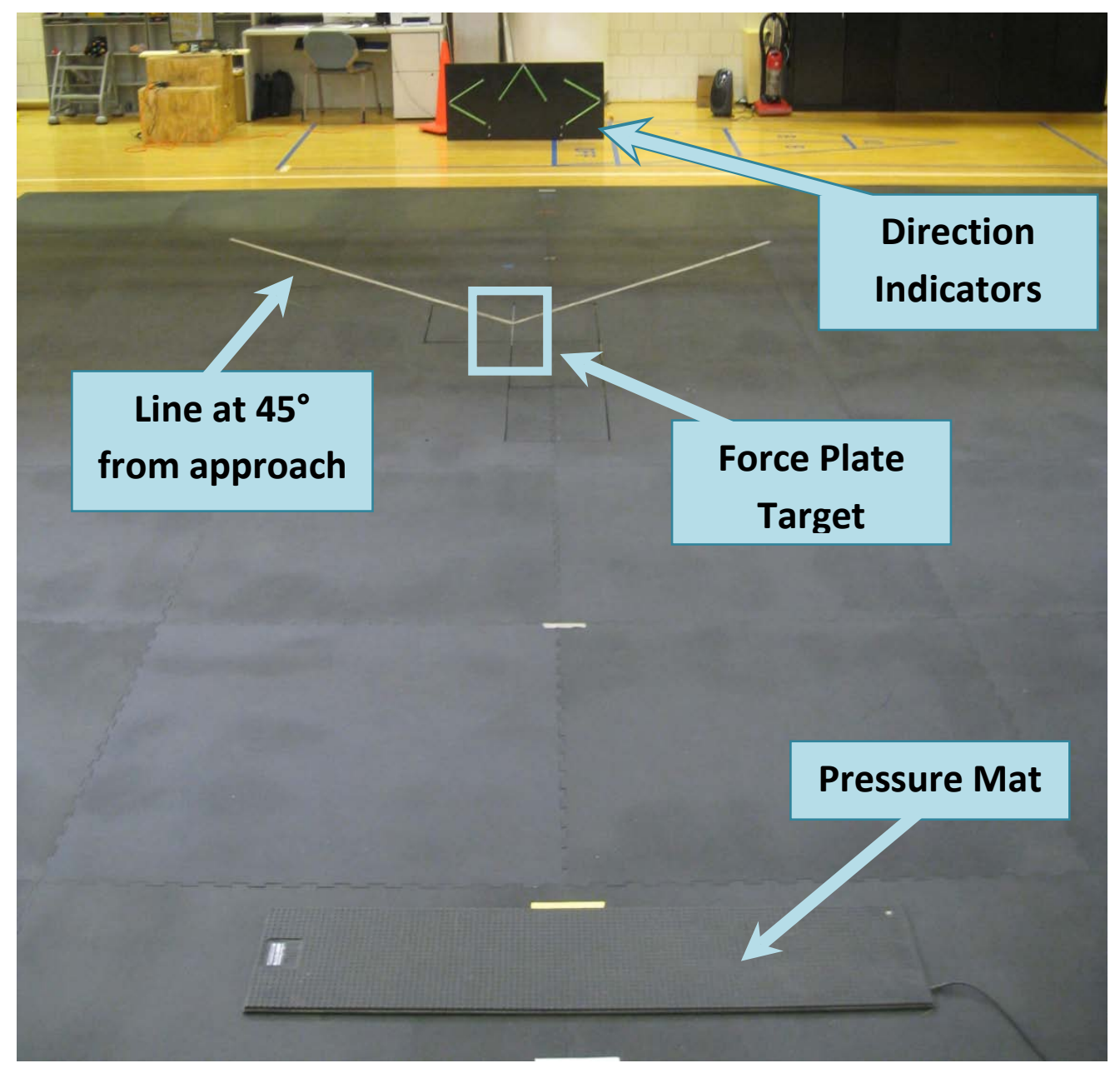

Figure 1: Unancipated run-to-cut set-up. Subject started on the pressure sensitive mat, took three steps at a self-selected jog pace before planting their fourth step within the force plate target area. A direction indicator arrow would illuminate before the plant indicating whether to perform a sidestep cut or continue straight. Right and left foot plants are possible with this set-up.

Knee Moments: The point-cluster technique [13] (Figure 2), as well as custom Vicon BodyBuilder [Vicon; Oxford, UK] and MATLAB [MathWorks, Inc., Natick, MA] scripts, were used to calculate kinematics and kinetics of the lower extremities curing the cut. Marker data was collected using 8 Vicon MX-F40 cameras at 300 Hz. Peak knee abduction and peak tibial internal rotation moments during the weight acceptance phase of the cut were estimated. Weight 
acceptance was defined as being between initial contact and peak knee flexion. Knee moments were normalized by body weight and height.
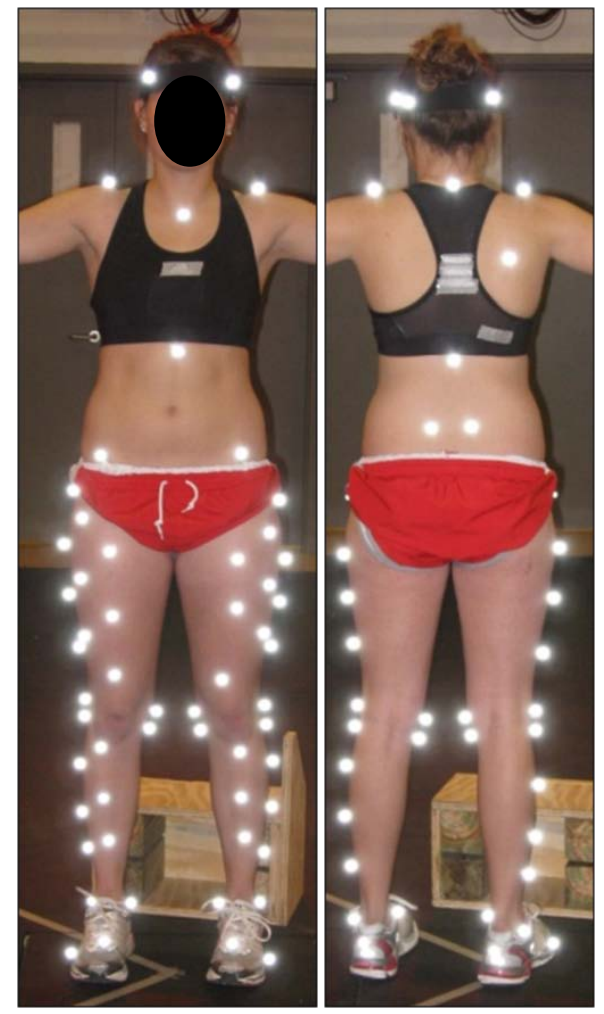

Figure 2: Anterior (left) and posterior ( right) views of the lower body Point-Cluster marker set combined with upper body Plug-In Gait marker set.

Torso Angles: Upper body markers based on the Plug-In Gait marker set [14] (Figure 2) were used to determine three torso angles (Figure 3). Again, marker data was collected at $300 \mathrm{~Hz}$ using 8 Vicon MX-F40 cameras. First, torso outside tilt was defined as the angle the torso deviated from vertical, with positive being a torso lean away from the direction of cutting. Peak outside tilt was found during the weight acceptance phase of the cut which was between foot strike and peak knee flexion. Two angles between the torso and the ground reaction force (GRF) were also calculated. The torso midline was established as the vector from the midpoint of the 
lower torso markers (sternum and T10) to the midpoint of the upper torso markers (clavicle and C7). The torso GRF-shoulders angle was defined as the angle between the GRF and the torso midline, in the plane containing the GRF and the shoulder markers. The torso GRF-pelvis angle was determined in a similar way, but used the plane containing the GRF and the midpoints of the right and left pelvic markers. Maximum torso-GRF angles were found during weight acceptance when the vertical GRF was greater than $10 \%$ of the subject's weight. Angles between the torso and GRF were chosen as the GRF is the decelerating force during the weight acceptance phase of the maneuver, when ACL injury occurs. Because previous studies have implicated lateral trunk position and control as ACL injury risk factors [12, 15], we examined frontal plane torso angles. Due to the relative rotation between the shoulders and the pelvis during a cutting task we chose to project the angle of the torso onto two frontal planes, one defined using the shoulders and the other using the pelvis, to examine the relationship between lateral trunk angles and knee moments. 


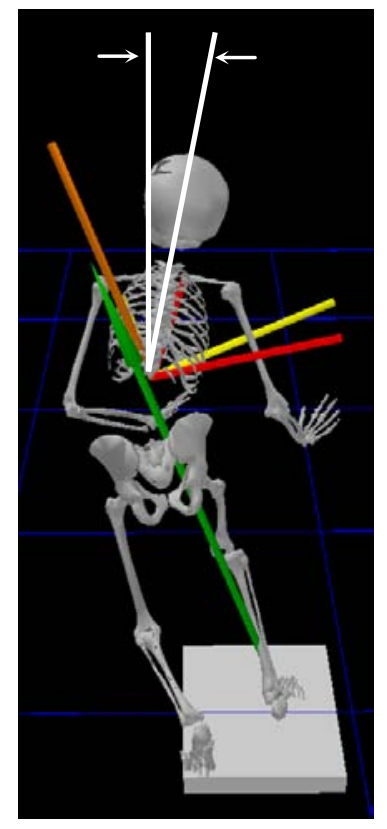

Figure 3: Posterior view of a subject performing the unanticipated run-to-cut maneuver, planting on their dominant limb and changing directions to the left. Both the plane containing the GRF and the shoulder markers (red and orange axes) and the plane containing the GRF and the midpoint of the right and left pelvic markers (yellow and orange axes) are depicted. The orange vector is common to both planes and is parallel to the GRF (green). Torso lean away from the GRF and vertical (white) can be seen as well as the lag in upper torso rotation relative to the pelvis.

Statistics: Knee abduction moments and torso GRF-pelvis angles were log transformed to meet normality assumptions for the statistical models. Tibial internal rotation moments, torso GRFshoulders angles, and outside tilt angles met normality assumptions without being log transformed. Linear mixed models were used to study the association between knee moments (Y) and torso angles (X). Peak knee abduction moment and peak tibial internal rotation moment were evaluated against each torso angle separately. Approach speed, cutting angle, and gender were considered as covariates in the models. A compound symmetry covariance structure was chosen to account for the correlation between the knee moments from the three trials of each subject. 


\section{RESULTS}

The results of the mixed effects models are summarized in Table 1 below. Significant positive associations were found between the log transformed peak knee abduction moment [ $\log (\mathrm{pKAM})]$ and outside tilt angle $(\mathrm{p}=0.009)$ and torso GRF-shoulders angle $(\mathrm{p}=0.029)$. Significant negative associations were found between tibial internal rotation moment (pTIRM) and outside tilt angle ( $\mathrm{p}=0.021)$. There was no evidence for a significant association between other variables of interest.

Table 1: Slope and p-values for interactions between normalized knee moments [\%BW*ht] and torso angles $\left[{ }^{\circ}\right]$ during a $45^{\circ}$ unanticipated run-to-cut maneuver. Bold values indicate $\mathbf{p} \leq \mathbf{0 . 0 5}$.

\begin{tabular}{|c|c|c|c|}
\hline & Angle & Slope & p-value \\
\hline \multirow{3}{*}{$\begin{array}{l}\widehat{\sum} \\
\text { 음 } \\
\text { 응 } \\
\underline{0}\end{array}$} & outside tilt & 0.04 & 0.009 \\
\hline & log torso GRF-pelvis & 0.625 & 0.146 \\
\hline & torso GRF-shoulders & 0.046 & 0.029 \\
\hline \multirow{3}{*}{ 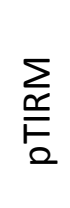 } & outside tilt & -0.025 & 0.021 \\
\hline & log torso GRF-pevis & -0.61 & 0.032 \\
\hline & torso GRF-shoulders & -0.016 & 0.72 \\
\hline
\end{tabular}

\section{DISCUSSION}

The results of this study supported our hypothesis concerning knee abduction moment, but did not support our hypothesis concerning tibial internal rotation moment. Significant positive associations between $\log (\mathrm{pKAM})$ and outside tilt and torso GRF-shoulders angles suggest that as the torso moves away from the cutting direction peak knee abduction moment increases. Significant negative associations between PTIRM and outside tilt and torso GRF- 
pelvis angles would suggest that as the torso moves away from the cutting direction peak tibial internal rotation moment decreases. These results are in direct conflict with each other. Because the knee abduction moments were log transformed to meet normality assumptions of the model, it is not possible to use the calculated slopes to determine whether on balance, the increase in knee abduction moment is counteracted by the decrease in tibial internal rotation moment.

The significant positive associations between $\log (\mathrm{pKAM})$ and torso angles indicate that an increased lean of the torso away from the cutting direction increases peak knee abduction moments. This effect is expected, because deviation of the torso from vertical causes the GRF to project more lateral of the knee, thereby increasing knee abduction moments (Figure 2). These results support the conclusions of Hewett et al. who examined videos of athletes performing landing and cutting maneuvers and found that females exhibited greater lateral trunk motion during ACL injury than control females during similar landing and cutting tasks [15]. 


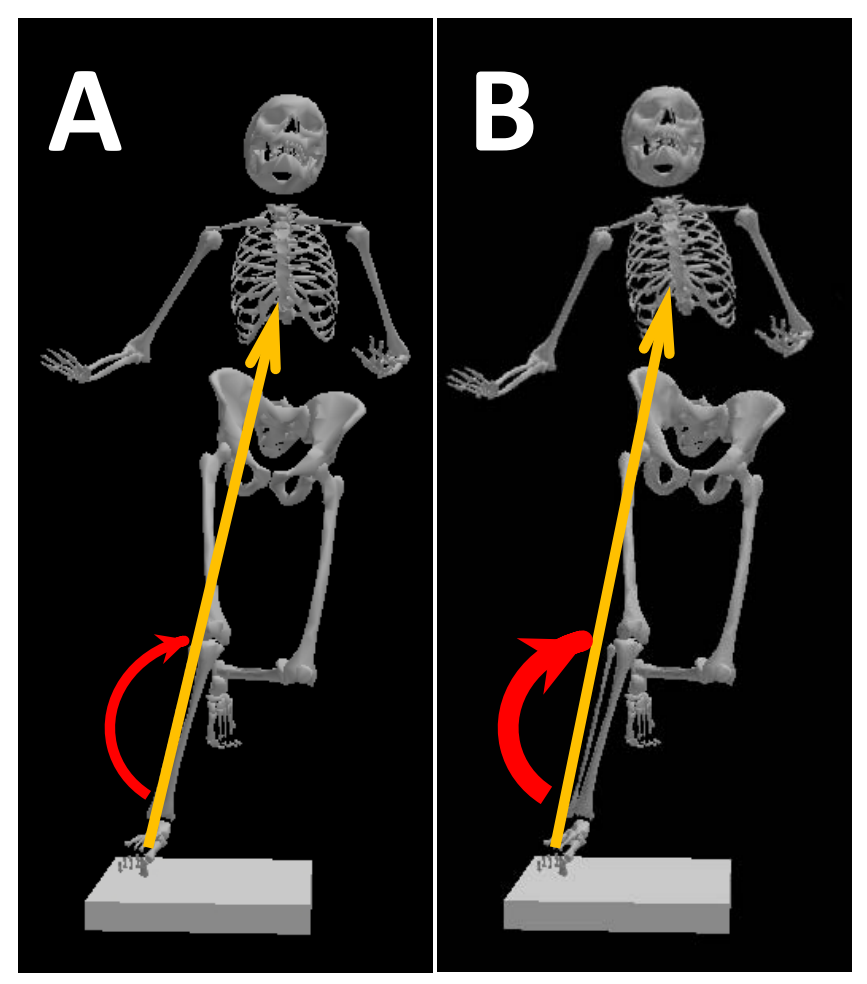

Figure 4: Subject planting with right foot and cutting to his/her left. A) Minimal torso lean away from the cutting direction. B) Increased torso lean projects the GRF (orange) more lateral of the knee and increases knee abduction moment (red).

The design of this study does not enable us to explain the increased torso lean in some subjects. One possible explanation is a lack of control of the trunk. In this scenario the pelvis and lower extremities progress toward the cutting direction, but the trunk musculature does not activate in such a way to keep the torso in line with lower extremity. Without that activation, the torso could lag behind the pelvis, resulting in torso lean away from the cutting direction. Another possibility is that the subject is utilizing an active control strategy. In this scenario the subject would be activating the musculature of the trunk in an effort to pull the torso away from the cutting direction. It could be that the subject is trying to counterbalance the lower body which is moving in the direction of the cut by moving the torso in the opposite direction. Subjects could also be employing this active control strategy in an effort to maximize power generation 
by coiling the torso away from the cut direction before springing it toward the direction of the cut. When considering the positive correlation between torso angles and knee abduction moments, as well as the findings of Hewett et al. [15], either scenario could be increasing ACL injury risk. Future studies should examine the root cause of the increased trunk angles using EMG or another means that quantifies trunk muscle activation.

\section{CONCLUSIONS}

This study observed an association between trunk lean and knee loading, providing evidence of a direct mechanical connection between dynamic trunk control and known risk factors for ACL injury. Further work is necessary to fully characterize the mechanical relationship between movement and control of the trunk and loading on the knee, but in the future it may be possible to design exercise programs that target both the trunk and lower extremities to reduce ACL injury risk.

\section{ACKNOWLEDGEMENTS}

Again I would like to thank my advisor, Ajit Chaudhari, PhD, and Jeff Pan, $\mathrm{PhD}$ for their significant contributions to this work. I would also like to gratefully acknowledge the financial support from the OSU Sports Medicine Initiative Grants Program and the NFL Charities Foundation. 


\section{REFERENCES}

1. Hewett, T.E., et al., The effect of neuromuscular training on the incidence of knee injury in female athletes: A prospective study. American Journal of Sports Medicine, 1999. 27(6(c) 2002 Inst. For Sci. Info): p. 699-706.

2. Freedman, K.B., et al., Anterior Cruciate Ligament Injury and Reconstruction Among University Students. Clinical Orthopaedics and Related Research, 1998. 356.

3. Ruiz, A.L., M. Kelly, and R.W. Nutton, Arthroscopic ACL reconstruction: a 5-9 year follow-up. The Knee, 2002. 9(3): p. 197-200.

4. Griffin, L.Y., et al., Noncontact Anterior Cruciate Ligament Injuries: Risk Factors and Prevention Strategies. J Am Acad Orthop Surg, 2000. 8(3): p. 141-150.

5. Boden, B.P., et al., Mechanisms of anterior cruciate ligament injury. Orthopedics, 2000. 23(6): p. 573-578.

6. McNair, P.J., R.N. Marshall, and J.A. Matheson, Important features associated with acute anterior cruciate ligament injury. N Z Med J, 1990. 103(901): p. 537-9.

7. Fleming, B.C., et al., The effect of weightbearing and external loading on anterior cruciate ligament strain. Journal of Biomechanics, 2001. 34(2): p. 163-170.

8. Kanamori, A., et al., The effect of axial tibial torque on the function of the anterior cruciate ligament: A biomechanical study of a simulated pivot shift test. Arthroscopy, 2002. 18(4): p. 394-398.

9. Shin, C.S., A.M. Chaudhari, and T.P. Andriacchi, Valgus Plus Internal Rotation Moments Increase ACL Strain More Than Either Alone. Medicine \& Science in Sports \& Exercise, 2011. Publish Ahead of Print.

10. Shin, C.S., A.M. Chaudhari, and T.P. Andriacchi, The effect of isolated valgus moments on ACL strain during single-leg landing: a simulation study. Journal of Biomechanics, 2009. 42(3): p. 280-5.

11. Hewett, T.E., et al., Biomechanical measures of neuromuscular control and valgus loading of the knee predict anterior cruciate ligament injury risk in female athletes: a prospective study. American Journal of Sports Medicine, 2005. 33(4): p. 492-501.

12. Zazulak, B.T., et al., Deficits in Neuromuscular Control of the Trunk Predict Knee Injury Risk: A Prospective Biomechanical-Epidemiologic Study. American Journal of Sports Medicine, 2007. 35(7): p. $1123-1130$.

13. Andriacchi, T.P., et al., A point cluster method for in vivo motion analysis: applied to a study of knee kinematics. Journal of Biomechanical Engineering, 1998. 120(6): p. 743-9.

14. Gutierrez, E.M., et al., Centre of mass motion during gait in persons with myelomeningocele. Gait and Posture, 2003. 18(2): p. 37-46.

15. Hewett, T.E., J.S. Torg, and B.P. Boden, Video analysis of trunk and knee motion during non-contact anterior cruciate ligament injury in female athletes: lateral trunk and knee abduction motion are combined components of the injury mechanism. British Journal of Sports Medicine, 2009. 43(6): p. 417-422. 